\title{
Factoring analytic multivariate polynomials and non-standard Cauchy-Riemann conditions
}

\author{
Tomas Recio ${ }^{\mathrm{a}}$, J.-Rafael Sendra ${ }^{\mathrm{b}}$, \\ Luis-Felipe Tabera ${ }^{\mathrm{a} *}$ Carlos Villarino ${ }^{\mathrm{b}}$

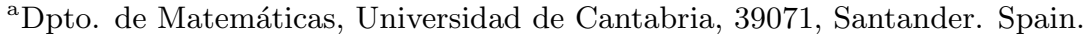 \\ ${ }^{\mathrm{b}}$ Dpto. de Físicas y Matemáticas, Universidad de Alcalá, 28871, Alcalá de Henares. \\ Spain.
}

\begin{abstract}
Motivated by previous work on the simplification of parametrizations of curves, in this paper we generalize the well known notion of analytic polynomial (a bivariate polynomial $P(x, y)$, with complex coefficients, which arises by substituting $z \rightarrow x+$ i $y$ on a univariate polynomial $p(z) \in \mathbb{C}[z]$, i.e. $p(z) \rightarrow p(x+\mathrm{i} y)=P(x, y))$ to other finite field extensions, beyond the classical case of $\mathbb{R} \subset \mathbb{C}$. In this general setting we obtain different properties on the factorization, gcd's and resultants of analytic polynomials, which seem to be new even in the context of Complex Analysis. Moreover, we extend the well-known Cauchy-Riemann conditions (for harmonic conjugates) to this algebraic framework, proving that the new conditions also characterize the components of generalized analytic polynomials.

keywords: Cauchy-Riemann conditions, analytic polynomials, Hankel matrix, factorization
\end{abstract}

\section{Introduction}

The well known Cauchy-Riemann (in short: CR) equations provide necessary and sufficient conditions for a complex function $f(z)$ to be holomorphic (c.f. [2], [5]). One traditional framework to introduce the CR conditions is through the consideration of harmonic conjugates, $\{u(x, y), v(x, y)\}$, as the real and imaginary parts of a holomorphic function $f(z)$, after performing the substitution $z \rightarrow x+\mathrm{i} y$ (i denotes the imaginary unit), yielding $f(x+\mathrm{i} y)=u(x, y)+\mathrm{i} v(x, y)$.

*Corresponding author: tel: +34 942201431, fax: +34 942201402

Email addresses: tomas.recio@unican.es (Tomas Recio), rafael.sendra@uah.es (J.-Rafael Sendra), taberalf@unican.es (Luis-Felipe Tabera), carlos.villarino@uah.es (Carlos Villarino) URL: http://www.recio.tk (Tomas Recio), http://www2.uah.es/rsendra/ (J.-Rafael Sendra), http://personales.unican.es/taberalf/ (Luis-Felipe Tabera) 
The Cauchy-Riemann conditions are a cornerstone in Complex Analysis and an essential ingredient of its many applications to Physics, Engineering, etc.

In this paper, we will consider two different, but related, issues. First, we will generalize CR conditions by replacing the real/complex framework by some more general field extension and, second, we will address -in this new settingthe specific factorization properties of conjugate harmonic polynomials. Let us briefly describe our approach to both topics in what follows.

In this paper, we will consider two different, but related, issues. First, we will generalize $\mathrm{CR}$ conditions by replacing the real/complex framework by some more general field extension and, second, we will address -in this new settingthe specific factorization properties of conjugate harmonic polynomials. Let us briefly describe our approach to both topics in what follows.

An analytic polynomial (a terminology taken from popular textbooks in Complex Analysis, see e.g. [2]), is a bivariate polynomial $P(x, y)$, with complex coefficients, which arises by substituting $z \rightarrow x+\mathrm{i} y$ on a univariate polynomial $p(z) \in \mathbb{C}[z]$, i.e. $p(z) \rightarrow p(x+\mathrm{i} y)=P(x, y)$. As stated above, a goal of our paper deals with generalizing $C R$ conditions when suitably replacing the pair real/complex numbers by some other field extension. Remark that, in the formulation of the CR conditions, since we restrict ourselves to polynomials and rational functions, we can use the well known concept of formal partial derivative of a polynomial or rational function, as in [9], Ch. II, $\S 17$, without requiring the introduction of any topological concept or the idea of limit of a function at a point. For a simple example, take as base field $\mathbb{K}=\mathbb{Q}$ and then $\mathbb{K}(\alpha)$, with $\alpha$ such that $\alpha^{3}+2=0$. Then we will consider polynomials (or more complicated functions) $f(z) \in \mathbb{K}(\alpha)[z]$ and perform the substitution $z=x_{0}+x_{1} \alpha+x_{2} \alpha^{2}$, yielding $f\left(x_{0}+x_{1} \alpha+x_{2} \alpha^{2}\right)=u_{0}\left(x_{0}, x_{1}, x_{2}\right)+u_{1}\left(x_{0}, x_{1}, x_{2}\right) \alpha+u_{2}\left(x_{0}, x_{1}, x_{2}\right) \alpha^{2}$, where, $u_{i} \in \mathbb{K}\left[x_{0}, x_{1}, x_{2}\right]$. Finally, we will like to find the necessary and sufficient conditions on a collection of polynomials $\left\{u_{i}\left(x_{0}, x_{1}, x_{2}\right)\right\}_{i=0,1,2}$ to be, as above, the components of the expansion of a polynomial $f(z)$ in the given field extension.

More generally, suppose $\mathbb{K}$ is a characteristic zero field, $\overline{\mathbb{K}}$ is the algebraic closure of $\mathbb{K}$, and $\alpha$ is an algebraic element over $\mathbb{K}$ of degree $r+1$. In this context we proceed, first, generalizing the concept of analytic polynomial as follows (see also [1],[7], as well as Definition 1 below, for a more general, multivariate, definition): 
A polynomial $p\left(x_{0}, \ldots, x_{r}\right) \in \mathbb{K}(\alpha)\left[x_{0}, \ldots, x_{r}\right]$ is called $\alpha$-analytic if there exists a polynomial $f(z) \in \overline{\mathbb{K}}[z]$ such that

$$
f\left(x_{0}+x_{1} \alpha+\cdots+x_{r} \alpha^{r}\right)=p\left(x_{0}, \ldots, x_{r}\right) .
$$

We say that $f$ is the generating polynomial of $p$. An analytic polynomial can be uniquely written as

$$
p\left(x_{0}, \ldots, x_{r}\right)=u_{0}\left(x_{0}, \ldots, x_{r}\right)+u_{1}\left(x_{0}, \ldots, x_{r}\right) \alpha+\cdots+u_{r}\left(x_{0}, \ldots, x_{r}\right) \alpha^{r},
$$

where $u_{i} \in \mathbb{K}\left[x_{0}, \ldots, x_{r}\right]$. The polynomials $u_{i}$ are called the $\mathbb{K}$-components of $p\left(x_{0}, \ldots, x_{r}\right)$.

The main result in this setting is the following statement (and its generalization to an even broader setting) expressing non-standard CR conditions (see Definition 3 and Theorem 15):

Let $\left\{u_{0}, \ldots, u_{r}\right\}$ be the $\mathbb{K}$-components of an $\alpha$-analytic polynomial $p\left(x_{0}, \ldots, x_{r}\right)$. It holds that

$$
\left(\begin{array}{c}
\frac{\partial u_{i}}{\partial x_{0}} \\
\vdots \\
\frac{\partial u_{i}}{\partial x_{r}}
\end{array}\right)=H_{i} \cdot\left(\begin{array}{c}
\frac{\partial u_{0}}{\partial x_{0}} \\
\vdots \\
\frac{\partial u_{r}}{\partial x_{0}}
\end{array}\right), \quad i=0, \ldots, r
$$

where $H_{i}$ the Hankel matrix introduced in Section 3. And, conversely, if these equations hold among a collection of polynomials $u_{i}$, then they are the $\mathbb{K}$-components of an analytic polynomial.

As expected, the above statement gives, in the complex case, the well known $\mathrm{CR}$ conditions. In fact, let $\mathbb{K}=\mathbb{R}, \alpha=i$, and $P\left(x_{0}, x_{1}\right) \in \mathbb{C}\left[x_{0}, x_{1}\right]$ be an analytic polynomial. If $u_{0}, u_{1}$ are the real and imaginary parts of $P$, the above Theorem states that

$$
\left(\begin{array}{l}
\frac{\partial u_{0}}{\partial x_{0}} \\
\frac{\partial u_{0}}{\partial x_{1}}
\end{array}\right)=\left(\begin{array}{rr}
1 & 0 \\
0 & -1
\end{array}\right) \cdot\left(\begin{array}{l}
\frac{\partial u_{0}}{\partial x_{0}} \\
\frac{\partial u_{1}}{\partial x_{0}}
\end{array}\right),\left(\begin{array}{l}
\frac{\partial u_{1}}{\partial x_{0}} \\
\frac{\partial u_{1}}{\partial x_{1}}
\end{array}\right)=\left(\begin{array}{ll}
0 & 1 \\
1 & 0
\end{array}\right) \cdot\left(\begin{array}{l}
\frac{\partial u_{0}}{\partial x_{0}} \\
\frac{\partial u_{1}}{\partial x_{0}}
\end{array}\right)
$$

which is a matrix form expression of the classic CR equations:

$$
\frac{\partial u_{0}}{\partial x_{1}}=-\frac{\partial u_{1}}{\partial x_{0}}, \frac{\partial u_{1}}{\partial x_{1}}=\frac{\partial u_{0}}{\partial x_{0}}
$$

It might be interesting to remark that the square matrix, expressing the above non-standard CR conditions, is a Hankel matrix (see [6] or Chapter 7 in [8]), an ubiquitous companion of Computer Algebra practitioners.

A computational relevant context (and in fact our original motivation) of our work about generalized analytic polynomials is the following situation. Consider a rational function $f(\mathbf{z}) \in \mathbb{C}(\mathbf{z})$ in several complex variables and with complex coefficients, then perform the substitution $\mathbf{z}=\mathbf{x}+\mathbf{i} \mathbf{y}$ and compute the real and imaginary parts of the resulting analytic rational function 
$f(\mathbf{x}+\mathbf{i} \mathbf{y})=u(\mathbf{x}, \mathbf{y})+\mathrm{i} v(\mathbf{x}, \mathbf{y})$. These two rational functions in $\mathbb{R}(\mathbf{x}, \mathbf{y})$ involve, usually, quite huge expressions, so it is reasonable to ask if there is a possibility of simplifying them by canceling out some common factors of the involved numerators and denominators. Such functions appear quite naturally when working with complex parametrizations of curves (see [3], and [4] for parametrizations with coefficients over a more general algebraic extension), and the key to attempt showing that some time-consuming steps could be avoided is, precisely, the analysis of the potential common factors for the two numerators of $u, v$. Learning about factorization properties of harmonic polynomials is useful in this respect. In fact, as a consequence of our study we can prove here that the assertion $\operatorname{gcd}(\operatorname{numer}(u)$, numer $(v))=1$ holds under reasonable assumptions and also that, if a rational function $f(\mathbf{z})$ in prime (also called irreducible) form is given, then the standard way of obtaining $u$ and $v$ yields also rational functions in prime form, i.e. it cannot be further simplified. See subsection 2.2 for more details on this application to the reparametrization problem.

More generally, in this paper we study (see Section 2) the factorization properties of generalized analytic polynomials, showing, among other remarkable facts, that conjugate harmonic polynomials cannot have a common factor (see Corollary 7). This seems a quite fundamental (and interesting) result, but we were not able to find a reference about it in the consulted bibliography within the Complex Variables context, probably because it requires an algebraic approach which is usually missing in the traditional Complex Analysis framework. On the other hand, we can generalize this result (in subsections 2.2 and 2.3) from polynomials to other functions (several variables, germs of holomorphic functions at a point, entire functions), all of them having in common being elements of rings with some factorization properties.

For expository reasons, we have chosen to structure this paper differently from the way we have presented the introduction, starting, first (see subsection 2.1), by the notion of $\alpha$-analytic multivariate polynomials and studying their basic algebraic properties; in particular those concerning factorization, gcd's and resultants. Then, in the last Section 3, we present the generalization of CR conditions to this new setting and we show that they (the new conditions) characterize components of $\alpha$-analytic multivariate polynomials (cf. Theorem 15). Moreover, as in the classical Complex Variables context, we can deduce again, from this non-standard CR conditions, some important properties of analytic polynomials (cf. Theorem 18).

\section{$2 \alpha$-Analyticity}

In this section we introduce the notion of $\alpha$-analyticity in different contexts, and we study its basic algebraic properties. 


\section{$2.1 \quad \alpha$-Analytic Multivariate Polynomials}

We start with the concept of $\alpha$-analytic polynomial. Let $\mathbb{K}$ be a characteristic zero field, $\overline{\mathbb{K}}$ its algebraic closure. Let $\alpha$ be an algebraic element of degree $r+1$ over $\mathbb{K}$ and let $n \geq 1$, a natural number. Let us introduce $r+1$ different tuples $\mathbf{x}_{i}=\left(x_{i 1}, \ldots, x_{i n}\right), 0 \leq i \leq r$, each one with $n$ different variables, thus, involving in total $(r+1) \times n$ variables. When adding these tuples, or multiplying them by constants $c \in \overline{\mathbb{K}}$, we will always perform the operations component-wise, i.e. $\mathbf{x}_{i}+\mathbf{x}_{j}=\left(x_{i 1}+x_{j 1}, \ldots, x_{i n}+x_{j n}\right), c \cdot \mathbf{x}_{i}=\left(c \cdot x_{i 1}, \ldots, c \cdot x_{i n}\right)$. Denote by $\mathbf{X}$ the tuple $\left(\mathbf{x}_{0}, \ldots, \mathbf{x}_{r}\right)$ and by $\mathbf{z}=\left(z_{1}, \ldots, z_{n}\right)$ a set of $n$ variables.

Definition 1. A polynomial $p(\mathbf{X})=p\left(\mathbf{x}_{0}, \ldots, \mathbf{x}_{r}\right) \in \mathbb{K}(\alpha)[\mathbf{X}]$ is called $(\alpha)-$ analytic if there exists a polynomial $f(\mathbf{z})=f\left(z_{1}, \ldots, z_{n}\right) \in \overline{\mathbb{K}}[\mathbf{z}]$ such that

$$
f\left(\mathbf{x}_{0}+\alpha \mathbf{x}_{1}+\cdots+\alpha^{r} \mathbf{x}_{r}\right)=p(\mathbf{X}) .
$$

In other words, $p(\mathbf{X})$ is the polynomial obtained replacing, in $f(\mathbf{z})$, each of the $z_{1}, \ldots, z_{n}$ variables by its "expansion" in terms of powers of $\alpha$, as follows:

$$
f\left(\sum_{i=0}^{r} \alpha^{i} x_{i 1}, \sum_{i=0}^{r} \alpha^{i} x_{i 2}, \ldots, \sum_{i=0}^{r} \alpha^{i} x_{i n}\right)=p\left(\mathbf{x}_{0}, \ldots, \mathbf{x}_{r}\right)
$$

where $x_{i j}, 0 \leq i \leq r, 1 \leq j \leq n$, are new variables. Notice that, for each $i$, $\mathbf{x}_{i}$ collects the $n$ variables involved in expressing the $i$-th power of $\alpha$ for the expansion of the different $z_{j}$ 's.

We say that $f(\mathbf{z})$ is a generating polynomial of $p(\mathbf{X})$ (but see Lemma 1 below, showing, in particular, that the generating polynomial is unique). If the fixed number of variables within each $\mathbf{x}_{i}$ is $n>1$, we say that the analytic polynomial is multivariate. An analytic polynomial can be uniquely written as

$$
p(\mathbf{X})=u_{0}(\mathbf{X})+u_{1}(\mathbf{X}) \alpha+\cdots+u_{r}(\mathbf{X}) \alpha^{r},
$$

where $u_{i} \in \mathbb{K}[\mathbf{X}]$. The polynomials $u_{i}$ are called the $\mathbb{K}$-components of $p(\mathbf{X})$.

Example 1. Let $\mathbb{K}=\mathbb{Q}, \alpha^{3}=5$, thus $r+1=3$, let $n=2$ and $f\left(z_{1}, z_{2}\right)=$ $z_{1} z_{2}+\alpha z_{2}^{2}+1$. Then $\mathbf{x}_{0}=\left(x_{01}, x_{02}\right), \mathbf{x}_{1}=\left(x_{11}, x_{12}\right), \mathbf{x}_{2}=\left(x_{21}, x_{22}\right)$. The $\alpha$-analytic polynomial generated by $f$, using formula (1) above, is:

$$
\begin{gathered}
p\left(\mathbf{x}_{0}, \mathbf{x}_{1}, \mathbf{x}_{2}\right)=f\left(x_{01}+\alpha x_{11}+\alpha^{2} x_{21}, x_{02}+\alpha x_{12}+\alpha^{2} x_{22}\right)= \\
=\left(x_{01}+\alpha x_{11}+\alpha^{2} x_{21}\right)\left(x_{02}+\alpha x_{12}+\alpha^{2} x_{22}\right)+\alpha\left(x_{02}+\alpha x_{12}+\alpha^{2} x_{22}\right)^{2}+1= \\
=p\left(x_{01}, x_{02}, x_{11}, x_{12}, x_{21}, x_{22}\right)
\end{gathered}
$$

Notice that the generating polynomial is not constrained, in principle, to belong to $\mathbb{K}(\alpha)[\mathbf{z}]$, but, more generally, it could be any element of $\overline{\mathbb{K}}[\mathbf{z}]$. It turns out that this is not possible, see Corollary 3 . The following result gives a simple criterion to decide whether a polynomial is analytic. 
Lemma 1 (Characterization of $\alpha$-analytic polynomials). A polynomial $p(\mathbf{X}) \in$ $\mathbb{K}(\alpha)[\mathbf{X}]$ is analytic if and only if

$$
p\left(\mathbf{x}_{0}+\alpha \mathbf{x}_{1}+\cdots+\alpha^{r} \mathbf{x}_{r}, \mathbf{0}, \ldots, \mathbf{0}\right)=p(\mathbf{X})
$$

if and only if for any (equivalently, all) $j, 1 \leq j \leq r$,

$$
p(\mathbf{X})=p\left(\mathbf{0}, \ldots, \mathbf{0}, \sum_{i=0}^{r} \alpha^{i-j} \mathbf{x}_{i}, \mathbf{0}, \ldots, \mathbf{0}\right),
$$

where the sum appears at the position of the tuple $\mathbf{x}_{j}$. Furthermore, in the affirmative case, there is a unique generating polynomial of $p(\mathbf{X})$ and it is $p(\mathbf{z}, \mathbf{0}, \ldots, \mathbf{0})$ or, equivalently, $p\left(\mathbf{0}, \ldots, \mathbf{0}, \mathbf{z} / \alpha^{i}, \mathbf{0}, \ldots, \mathbf{0}\right), 1 \leq i \leq r$.

Proof. If $p\left(\mathbf{x}_{0}+\alpha \mathbf{x}_{1}+\cdots+\alpha^{r} \mathbf{x}_{r}, \mathbf{0}, \ldots, \mathbf{0}\right)=p(\mathbf{X})$, then $p(\mathbf{X})$ is the analytic polynomial generated by $p(\mathbf{z}, \mathbf{0}, \ldots, \mathbf{0})$. Conversely, if $p(\mathbf{X})$ is analytic and $f(\mathbf{z})$ is any generating polynomial of $p(\mathbf{X})$ then we have the polynomial identity (1)

$$
f\left(\sum_{i=0}^{r} \alpha^{i} x_{i 1}, \ldots, \sum_{i=0}^{r} \alpha^{i} x_{i n}\right)=p(\mathbf{X})
$$

Substituting $x_{i j}=0$ for $1 \leq i \leq r, 1 \leq j \leq n$, we get

$$
f\left(x_{01}, \ldots, x_{0 n}\right)=p\left(\mathbf{x}_{0}, \mathbf{0}, \ldots, \mathbf{0}\right)
$$

and, hence, the generating polynomial $f$ is uniquely determined by $p$, yielding $f(\mathbf{z})=p(\mathbf{z}, \mathbf{0}, \ldots, \mathbf{0})$. Now, if we substitute $x_{0 i}=\sum_{j=0}^{r} \alpha^{j} x_{j i}$ in $(2)$, we get

$$
p\left(\sum_{i=0}^{r} \alpha^{i} \mathbf{x}_{i}, \mathbf{0}, \ldots, \mathbf{0}\right)=f\left(\sum_{i=0}^{r} \alpha^{i} \mathbf{x}_{i}\right)=p(\mathbf{X}) .
$$

For any other index $1 \leq i \leq r$, the proof is similar.

A direct and very useful consequence of this lemma is the following result.

Corollary 2. Let $p(\mathbf{X})$ be $\alpha$-analytic generated by $f(\mathbf{z})$. Then $p$ is constant if and only if $f$ is constant.

We observe that the set of $\alpha$-analytic polynomials over $\mathbb{K}(\alpha)$ is a subring of $\mathbb{K}(\alpha)[\mathbf{X}]$. We denote it by $\mathbb{A}_{\alpha}[\mathbf{X}]$. Moreover, the set of its generating polynomials is a subring of $\overline{\mathbb{K}}[\mathbf{z}]$. We denote it by $\mathbb{G}_{\alpha}[\mathbf{z}]$. Now, in Definition 1 we have introduced the generating polynomials as polynomials with coefficients in $\overline{\mathbb{K}}$. However, from Lemma 1 one deduces that their coefficients are in $\mathbb{K}(\alpha)$. Thus, we get the following equality.

Corollary 3. $\mathbb{G}_{\alpha}[\mathbf{z}]=\mathbb{K}(\alpha)[\mathbf{z}]$.

As consequence of Lemma 1, we also deduce the following property that, in particular, implies that $\mathbb{A}_{\alpha}[\mathbf{X}]$ is a proper subring of $\mathbb{K}(\alpha)[\mathbf{X}]$. 
Corollary 4. $(\mathbb{K}[\mathbf{X}] \backslash \mathbb{K}) \cap \mathbb{A}_{\alpha}[\mathbf{X}]=\emptyset$, i.e. there are no analytic polynomials with coefficients in $\mathbb{K}$ other than constants.

Proof. Let $p(\mathbf{X}) \in \mathbb{A}_{\alpha}[\mathbf{X}] \cap \mathbb{K}[\mathbf{X}]$ be non-constant. Let $f(\mathbf{z})$ be its generator. By Lemma $1, f \in \mathbb{K}[\mathbf{z}]$. First we prove that there exists $\bar{\gamma} \in \mathbb{K}(\alpha)^{n}$ such that $f(\bar{\gamma}) \notin \mathbb{K}$. From there, writing $\bar{\gamma}$ as $\bar{\gamma}=\bar{\gamma}_{0}+\cdots+\alpha^{r} \bar{\gamma}_{r}$, with $\overline{\gamma_{i}} \in \mathbb{K}^{n}$, we get that $f(\bar{\gamma})=p\left(\bar{\gamma}_{0}, \ldots, \bar{\gamma}_{r}\right) \in \mathbb{K}$, which is a contradiction. Since $f$ is not constant (see Corollary 2), $f$ depends on at least one variable $z_{i}$; say w.l.o.g. on $z_{n}$. We express $f$ as a univariate polynomial in $z_{n}$ : $f(\mathbf{z})=A_{m}\left(z_{1}, \ldots, z_{n-1}\right) z_{n}^{m}+\cdots+A_{0}\left(z_{1}, \ldots, z_{n-1}\right)$, with $m>0$. Now we take $a_{1}, \ldots, a_{n-1} \in \mathbb{K}$ such that $A_{m}\left(a_{1}, \ldots, a_{n-1}\right) \neq 0$. Then, $f\left(a_{1}, \ldots, a_{n-1}, z_{n}\right) \in$ $\mathbb{K}\left[z_{n}\right]$ and is not constant. In this situation is clear that there exist $a_{n} \in \mathbb{K}(\alpha)$ such that $f\left(a_{1}, \ldots, a_{n-1}, a_{n}\right) \in \mathbb{K}(\alpha) \backslash \mathbb{K}$. Finally, take $\bar{\gamma}=\left(a_{1}, \ldots, a_{n}\right)$.

The following result states that the ring of analytic polynomials and the ring of generating polynomials are isomorphic.

Theorem 5. $\mathbb{A}_{\alpha}[\mathbf{X}]$ is $\mathbb{K}(\alpha)$-isomorphic to $\mathbb{G}_{\alpha}[\mathbf{z}]$.

Proof. We consider the map $\phi: \mathbb{G}_{\alpha}[\mathbf{z}] \rightarrow \mathbb{A}_{\alpha}[\mathbf{X}]$ such that $\phi(f(\mathbf{z}))=f\left(\mathbf{x}_{0}+\right.$ $\left.\alpha \mathbf{x}_{1}+\cdots+\alpha^{r} \mathbf{x}_{r}\right)$. Clearly, $\phi$ is a ring homomorphism. Moreover, Lemma 1 ensures that $\phi$ is onto and injective since zero is the (unique) generating polynomial of zero. Furthermore, the restriction of $\phi$ to $\mathbb{K}(\alpha)$ is the identity map.

Applying Theorem 5, we derive properties on factorization, gcd's, and resultants of analytic polynomials. First we observe that, since $\mathbb{G}_{\alpha}[\mathbf{z}]$ is a unique factorization domain (UFD), and since $\phi$ (the $\mathbb{K}(\alpha)$-isomorphism introduced in the proof of Theorem 5) preserves constants, we have the following Corollary.

Corollary 6. $\mathbb{A}_{\alpha}[\mathbf{X}]$ is a unique factorization domain

Again, Theorem 5 can be used to relate the factors of an analytic polynomial to the factors of its generator. More precisely, one has the next result.

Corollary 7 (Factorization properties). Let $p(\mathbf{X}) \in \mathbb{A}_{\alpha}[\mathbf{X}]$ be generated by $f(\mathbf{z}) \in \mathbb{G}_{\alpha}[\mathbf{z}]$. It holds that

1. $p(\mathbf{X})$ is irreducible in $\mathbb{A}_{\alpha}[\mathbf{X}]$ iff $p(\mathbf{X})$ is irreducible in $\mathbb{K}(\alpha)[\mathbf{X}]$.

2. $p(\mathbf{X})$ is irreducible in $\mathbb{K}(\alpha)[\mathbf{X}]$ iff $f(\mathbf{z})$ is irreducible in $\mathbb{K}(\alpha)[\mathbf{z}]$.

3. $f(\mathbf{z})=f_{1}(\mathbf{z})^{n_{1}} \cdots f_{s}(\mathbf{z})^{n_{s}}$ is an irreducible factorization of $f$ in $\mathbb{K}(\alpha)[\mathbf{z}]$ iff $p(\mathbf{X})=f_{1}\left(\mathbf{x}_{0}+\cdots+\alpha^{r} \mathbf{x}_{r}\right)^{n_{1}} \cdots f_{s}\left(\mathbf{x}_{0}+\cdots+\alpha^{r} \mathbf{x}_{r}\right)^{n_{s}}$ is an irreducible factorization of $p$ in $\mathbb{K}(\alpha)[\mathbf{X}]$.

4. $p(\mathbf{X})$ has no factor in $\mathbb{K}[\mathbf{X}]$.

5. Let $\left\{u_{i}(\mathbf{X}), 0 \leq i \leq r\right\}$ be the $\mathbb{K}$-components of $p(\mathbf{X})$, so that $p=\sum_{i=0}^{r} u_{i} \alpha^{i}$. Then, $\operatorname{gcd}\left(u_{0}, \ldots, u_{r}\right)=1$. 
Proof. (1) The right-left implication is clear. Conversely, let $p$ be irreducible as an element of $\mathbb{A}_{\alpha}[\mathbf{X}]$. Now, assume that $p=A B$, where $A, B$ are non-constant polynomials in $\mathbb{K}(\alpha)[\mathbf{X}]$. Since $p$ is analytic, by Lemma $1, p(\mathbf{X})=p\left(\mathbf{x}_{0}+\cdots+\right.$ $\left.\alpha^{r} \mathbf{x}_{r}, \mathbf{0}, \ldots, \mathbf{0}\right)=A\left(\mathbf{x}_{0}+\cdots+\alpha^{r} \mathbf{x}_{r}, \mathbf{0}, \ldots, \mathbf{0}\right) B\left(\mathbf{x}_{0}+\cdots+\alpha^{r} \mathbf{x}_{r}, \mathbf{0}, \ldots, \mathbf{0}\right)$. Now observe that both $A\left(\mathbf{x}_{0}+\cdots+\alpha^{r} \mathbf{x}_{r}, \mathbf{0}, \ldots, \mathbf{0}\right)$ and $B\left(\mathbf{x}_{0}+\cdots+\alpha^{r} \mathbf{x}_{r}, \mathbf{0}, \ldots, \mathbf{0}\right)$ are analytic polynomials, $A\left(\mathbf{x}_{0}+\cdots+\alpha^{r} \mathbf{x}_{r}, \mathbf{0}, \ldots, \mathbf{0}\right), B\left(\mathbf{x}_{0}+\cdots+\alpha^{r} \mathbf{x}_{r}, \mathbf{0}, \ldots, \mathbf{0}\right) \in$ $\mathbb{A}_{\alpha}[\mathbf{X}]$. Thus, since $p$ is irreducible as analytic polynomial, one of them has to be constant, say $A\left(\mathbf{x}_{0}+\cdots+\alpha^{r} \mathbf{x}_{r}, \mathbf{0}, \ldots, \mathbf{0}\right)=\lambda \in \mathbb{K}(\alpha)$. Then,

$$
A(\mathbf{X}) B(\mathbf{X})=p(\mathbf{X})=\lambda B\left(\mathbf{x}_{0}+\cdots+\alpha^{r} \mathbf{x}_{r}, \mathbf{0}, \ldots, \mathbf{0}\right)
$$

Finally, since $A(\mathbf{X})$ is not constant, this implies that the total degree of $B\left(\mathbf{x}_{0}+\right.$ $\left.\cdots+\alpha^{r} \mathbf{x}_{r}, \mathbf{0}, \ldots, \mathbf{0}\right)$ is greater than the total degree of $B(\mathbf{X})$, which is impossible.

Statements (2) and (3) follow from (1) and Theorem 5. Statement (4) is a consequence of (1) and Corollary 4. Statement (5) follows from (4), since $\operatorname{gcd}\left(u_{0}, \ldots, u_{r}\right)$ is a factor of $p$ with coefficients in $\mathbb{K}$.

Similarly, one may relate the gcd of several analytic polynomials to the gcd of their generators.

Corollary 8 (Gcd formula). Let $p_{1}, \ldots, p_{s} \in \mathbb{A}_{\alpha}[\mathbf{X}]$ be $\alpha$-analytic polynomials generated by $f_{1}, \ldots, f_{s} \in \mathbb{G}_{\alpha}[\mathbf{z}]$, respectively. It holds that

$$
\operatorname{gcd}\left(p_{1}(\mathbf{X}), \ldots, p_{s}(\mathbf{X})\right)=\operatorname{gcd}\left(f_{1}(\mathbf{z}), \ldots, f_{s}(\mathbf{z})\right)\left(\mathbf{x}_{0}+\cdots+\alpha^{r} \mathbf{x}_{r}\right) .
$$

Finally, we study the computation of resultants of polynomials in $\mathbb{A}_{\alpha}[\mathbf{X}][w]$, i.e. of univariate polynomials with coefficients in the ring $\mathbb{A}_{\alpha}[\mathbf{X}]$. For this purpose, we will refer to the natural extension $\phi^{\star}: \mathbb{G}_{\alpha}[\mathbf{z}][w] \rightarrow \mathbb{A}_{\alpha}[\mathbf{X}][w]$ of the isomorphism $\phi: \mathbb{G}_{\alpha}[\mathbf{z}] \rightarrow \mathbb{A}_{\alpha}[\mathbf{X}]$ (introduced in the proof of Theorem 5) to these new polynomial rings. In this situation, one has the next corollary.

Corollary 9 (Resultant formula). Let $P_{1}, P_{2} \in \mathbb{A}_{\alpha}[\mathbf{X}][w]$ be generated, respectively, via $\phi^{\star}$, by $F_{1}, F_{2} \in \mathbb{G}_{\alpha}[\mathbf{z}][w]$. It holds that

$$
\operatorname{Resultant}_{w}\left(P_{1}, P_{2}\right)=\operatorname{Resultant}_{w}\left(F_{1}, F_{2}\right)\left(\mathbf{x}_{0}+\cdots+\alpha^{r} \mathbf{x}_{r}\right)
$$

Proof. Let $M$ be the Sylvester matrix associated to $P_{1}, P_{2}$, and let $N$ be the Sylvester matrix associated to $F_{1}, F_{2} . M$ is over $\mathbb{A}_{\alpha}[\mathbf{X}]$, and $N$ is over $\mathbb{G}_{\alpha}[\mathbf{z}]$. Therefore, since a determinant only involves additions and multiplications in the corresponding ring, the result follows using Theorem 5 .

\section{$2.2 \alpha$-Analytic Multivariate Rational Functions: An ap- plication}

As in the previous subsection and following the notation thereof, a rational function $A(\mathbf{X}) \in \mathbb{K}(\alpha)(\mathbf{X})$ will be called $(\alpha)$-analytic if there exists a rational function $B(\mathbf{z}) \in \overline{\mathbb{K}}(\mathbf{z})$ such that

$$
B\left(\mathbf{x}_{0}+\alpha \mathbf{x}_{1}+\cdots+\alpha^{r} \mathbf{x}_{r}\right)=A(\mathbf{X}) .
$$


We say that $B(\mathbf{z})$ is the generating rational function of $A$. If $n>1$, we say that the analytic rational function is multivariate.

In [3], a complete analysis for i-analytic univariate rational functions is given, in the context of a reparametrization problem for curves. Namely, given a rational curve parametrization with complex coefficients, the parametrization problem consists in deciding whether the curve can be also parametrized with real rational functions and, in the affirmative case, in computing a parameter change, that transforms the initial complex parametrization into a real one. Obviously, this context can be generalized to the case of rational parametrizations with coefficients in a finite algebraic extension, of a given ground field: here, the goal is also to simplify a given parametrization with algebraic coefficient to another one with coefficients over the ground field.

In this context (see, for more details, [1], [4]), the following situation happens. Let $f=a(\mathbf{z}) / b(\mathbf{z}) \in \mathbb{K}(\alpha)(\mathbf{z})$ be a rational function. Then, we want to compute the $\mathbb{K}$-components of $\phi(f) \in \mathbb{A}_{\alpha}(\mathbf{X})$ (where $\phi$ is the extension to rational functions of the isomorphism introduced in Theorem 5). One way to proceed is first apply the change of variables $p=a\left(\mathbf{x}_{0}+\alpha \mathbf{x}_{1}+\ldots+\alpha^{r} \mathbf{x}_{r}\right)$, $q=b\left(\mathbf{x}_{0}+\alpha \mathbf{x}_{1}+\ldots+\alpha^{r} \mathbf{x}_{r}\right)$, and then compute $Q$, the multiple of $q$ of smallest degree that has all its coefficients in $\mathbb{K}$. It is easy to verify that $Q$ is a divisor of the norm of $q$ for the extension $\mathbb{K}[\mathbf{X}] \subseteq \mathbb{K}[\mathbf{X}](\alpha)$. Let $R=Q / q \in \mathbb{K}(\alpha)[\mathbf{X}]$. It follows that $\phi(f)=p \cdot R / Q$ and the $\mathbb{K}$-components are $\phi(f)=\sum_{i=0}^{r}\left(u_{i} / Q\right) \alpha^{i}$, where $u_{i}$ are the $\mathbb{K}$-components of $p \cdot R$. This is a mathematically valid representation, but it is desirable for applications -such as the ones mentioned abovethat $\operatorname{gcd}\left(u_{0}, \ldots, u_{r}, Q\right)=1$. The interesting fact is that this is the case under natural assumptions.

Theorem 10. Assume that $\operatorname{gcd}(a, b)=1$ in the previous construction, then the above procedure to compute the $\mathbb{K}$-components of $f(\mathbf{z})=a(\mathbf{z}) / b(\mathbf{z})$ verifies that $\operatorname{gcd}\left(u_{0}, \ldots, u_{r}, Q\right)=1$

Proof. Note that, in general, $p \cdot R$ need not be $\alpha$-analytic, so we can not use Corollary 7 directly. Since $\operatorname{gcd}(a, b)=1$, then, by Corollary $8, \operatorname{gcd}(p, q)=1$ and $\operatorname{gcd}(p \cdot R, q \cdot R)=R$. If $c=\operatorname{gcd}\left(u_{i}, Q\right)$ then $c$ is a polynomial with coefficients over $\mathbb{K}$ and $c \mid R$, but $R$ does not have factors over $\mathbb{K}$. To prove this, let $d$ be a factor of $R$ with coefficients in $\mathbb{K}$ irreducible in $\mathbb{K}[\mathbf{X}]$. Then $d$ is also a factor of $Q$ and, by construction of $Q, \operatorname{gcd}(d, q) \neq 1$. Let $g$ be an irreducible factor of $\operatorname{gcd}(d, q)$ with coefficients in $\mathbb{K}(\alpha)[\mathbf{X}]$ and assume that $q=g^{l} q_{1}, \operatorname{gcd}\left(g, q_{1}\right)=1$. Let $G$ be the minimum multiple of $g$ with coefficients over $\mathbb{K}$. Then $G \mid d$, but $d$ is irreducible in $\mathbb{K}[\mathbf{X}]$, so $G=d$. It follows that $Q=d^{l} Q_{1}, \operatorname{gcd}\left(d, Q_{1}\right)=1$. But then, $R=Q / q$ does not have $g$ as a factor, so $d$ cannot be a factor of $R$.

\subsection{The case of germs and entire functions}

In this section we generalize the previous notions and results to germs of holomorphic functions and entire functions. Because these mathematical entities are defined only over the complex numbers, in this subsection we will consider always that $\mathbb{K}=\mathbb{R}$ and $\alpha=i=\sqrt{-1}$. Let $\mathbf{a}=\left(a_{1}+\mathrm{i} b_{1}, \ldots, a_{n}+\mathrm{i} b_{n}\right) \in \mathbb{C}^{n}$, 
$a_{j}, b_{j} \in \mathbb{R}, \mathbf{c}=\left(a_{1}, \ldots, a_{n}, b_{1}, \ldots, b_{n}\right) \in \mathbb{R}^{2 n} \subset \mathbb{C}^{2 n}, \mathbf{x}=\left(x_{1}, \ldots, x_{n}\right), \mathbf{y}=$ $\left(y_{1}, \ldots, y_{n}\right)$ and $\mathbf{z}=\left(z_{1}, \ldots, z_{n}\right)$. Furthermore, let $\mathcal{O}_{\mathbb{C}^{n}, \mathbf{a}}$ be the local ring of complex holomorphic germs at the point $\mathbf{a}$; recall that it is isomorphic to the ring of complex convergent power series centered at $\mathbf{a}$. Similar notation will be used to express other local rings of germs.

In this situation, if $\mathbf{f}(\mathbf{z}) \in \mathcal{O}_{\mathbb{C}^{n}, \mathbf{a}}$ we consider the complex holomorphic germ $\mathbf{p}(\mathbf{x}, \mathbf{y})=\mathbf{f}(\mathbf{x}+\mathbf{i} \mathbf{y}) \in \mathcal{O}_{\mathbb{C}^{2 n}, \mathbf{c}}$. Clearly, $\mathbf{p}(\mathbf{x}, \mathbf{y}) \in \mathcal{O}_{\mathbb{C}^{2 n}, \mathbf{c}}$ can always be expressed as:

$$
\mathbf{p}(\mathbf{x}, \mathbf{y})=\mathbf{u}(\mathbf{x}, \mathbf{y})+\mathrm{i} \mathbf{v}(\mathbf{x}, \mathbf{y})
$$

where $\mathbf{u}, \mathbf{v}$ are real analytic germs at $\mathbf{c}$ (the real and imaginary parts of $\mathbf{p}(\mathbf{x}, \mathbf{y})$ ). Thus, since the ring $\mathcal{O}_{\mathbb{C}^{2 n} \text {,c }}$ is a unique factorization domain, one can study factorization questions for $\mathbf{p}(\mathbf{x}, \mathbf{y})$. Furthermore, since the ring $\mathcal{O}_{\mathbb{R}^{2 n}, \mathbf{c}}$ of real analytic germs at $\mathbf{c} \in \mathbb{R}^{2 n}$ is also a unique factorization domain, one can consider the gcd of its elements; in particular the gcd of the germs $\mathbf{u}$ and $\mathbf{v}$. In this context, (multiplicative) units play the role of constants (in the polynomial case); they are the germs that do not vanish at $\mathbf{c}$. Then it is easy to show that $\mathbf{p}$ is a unit in $\mathcal{O}_{\mathbb{C}^{2 n}, \mathbf{c}}$ if and only if $\mathbf{f}$ is a unit in $\mathcal{O}_{\mathbb{C}^{n}, \mathbf{a}}$, if and only if either $\mathbf{u}$ or $\mathbf{v}$ are units in $\mathcal{O}_{\mathbb{R}^{2 n}, \mathbf{c}}$. Moreover, by the classical Cauchy-Riemann conditions, one has that $\mathbf{p}$ is constant if and only if $\mathbf{u}$ or $\mathbf{v}$ are constants.

Proposition 11. Let $\mathbf{p} \in \mathcal{O}_{\mathbb{C}^{2 n}, \mathbf{c}}$ be a nonconstant germ of the form $\mathbf{p}(\mathbf{x}, \mathbf{y})=$ $\mathbf{f}(\mathbf{x}+\mathbf{i y})$, for some $\mathbf{f}(\mathbf{z}) \in \mathcal{O}_{\mathbb{C}^{n}, \mathbf{a}}$. Then $\mathbf{p} \notin \mathcal{O}_{\mathbb{R}^{2 n}, \mathbf{c}}$. Moreover, it is not associated to any real-defined germ. That is, if $\mathbf{u} \in\left(\mathcal{O}_{\mathbb{C}^{2 n}, \mathbf{c}}\right)^{*}$ (the ring of units), then $\mathbf{u} \cdot \mathbf{p} \notin \mathcal{O}_{\mathbb{R}^{2 n}, \mathbf{c}}$.

Proof. If $\mathbf{p}$ is a real-defined germ $\mathbf{p} \in \mathcal{O}_{\mathbb{R}^{2 n}, \mathbf{c}}, \mathbf{p}(\mathbf{x}, \mathbf{y}) \in \mathbb{R}$ for all $(\mathbf{x}, \mathbf{y})$ in an open neighborhood $U \subseteq \mathbb{R}^{2 n}$ of $\mathbf{c}$. If this happens, then $\mathbf{f}(V) \subseteq \mathbb{R}$ for an open neighborhood $V \subseteq \mathbb{C}^{n}$ of a. But, from basic properties of analytic functions, f (and p) must be, then, constant functions, contradicting the hypothesis. For the second part, assume without loss of generality that $\mathbf{c}=(0, \ldots, 0)$. Let $r \cdot z_{1}^{a_{1}} \cdots z_{n}^{a_{n}}$ be a term of minimal degree in the power series expansion of $\mathbf{f}(\mathbf{z})$. In the power expansion of $\mathbf{p}$, we have some lowest degree terms such as $r \cdot x_{1}^{a_{1}} \ldots x_{n}^{a_{n}}$ and $\mathrm{i} a_{1} r \cdot y_{1} x_{1}^{a_{1}-1} x_{2}^{a_{2}} \cdots x_{n}^{a_{n}}$. If $\mathbf{u}$ is a unit of $\mathcal{O}_{\mathbb{C}^{2 n}, 0}$, then $\mathbf{u}(0)=b \neq 0$. Now, in the powers series expansion of $\mathbf{u} \cdot \mathbf{p}$ we get the coefficients $b \cdot r$ and $b \cdot \mathbf{i} a_{1} r$, which cannot be both real. This proves that $\mathbf{u} \cdot \mathbf{p}$ cannot be a real-defined germ.

We can now obtain a result analogous to Corollary 7 , but in the context of germs.

Proposition 12. Let $\mathbf{p} \in \mathcal{O}_{\mathbb{C}^{2 n}, \mathbf{c}}$ be as above, $\mathbf{p}(\mathbf{x}, \mathbf{y})=\mathbf{f}(\mathbf{x}+\mathbf{i y})$. Then

- $\mathbf{p}$ is irreducible if and only if $\mathbf{f}$ is irreducible.

- If $\mathbf{f}=f_{1}^{i_{1}} \cdots f_{s}^{i_{s}}$ is the irreducible factorization of $\mathbf{f}$ then $\mathbf{p}=f_{i}^{i_{1}}(\mathbf{x}+$ iy) $\cdots f_{s}^{i_{s}}(\mathbf{x}+\mathbf{i y})$ is the irreducible factorization of $\mathbf{p}$.

- $\mathbf{p}$ has no real-defined factor. 
Proof. Clearly $\mathbf{p}(\mathbf{x}, \mathbf{y})=\mathbf{p}(\mathbf{x}+\mathbf{i} \mathbf{y}, 0)=f(\mathbf{x}+\mathbf{i} \mathbf{y})$ and, if $\mathbf{f}$ is reducible, then $\mathbf{p}$ is reducible. Now, assume that $\mathbf{p}$ is reducible and $\mathbf{p}=A \cdot B, A$ and $B$ non-units. Then $\mathbf{f}(\mathbf{x}+\mathbf{i} \mathbf{y})=A(\mathbf{x}+\mathbf{i} \mathbf{y}, 0) B(\mathbf{x}+\mathbf{i} \mathbf{y}, 0)$. If $A(\mathbf{x}+\mathbf{i} \mathbf{y}, 0)$ were a unit, then $\mathbf{f}(\mathbf{x}+\mathbf{i} \mathbf{y})=\lambda B(\mathbf{x}+\mathbf{i} \mathbf{y}, 0)$, so the order of $\mathbf{p}$ at $\mathbf{c}$ equals the order of $B$ and the order of $A$ at $\mathbf{c}$ is zero. So $A$ would be a unit which is a contradiction. So $A(\mathbf{x}+\mathbf{i y}, 0)$ is not a unit and, by symmetry, $B(\mathbf{x}+\mathbf{i y})$ is neither a unit. It follows that $\mathbf{f}=A(\mathbf{x}+\mathbf{i} \mathbf{y}, 0) B(\mathbf{x}+\mathbf{i} \mathbf{y}, 0)$ is reducible.

The second item follows directly from the first one and the third item follows from the first item and Proposition 11.

Now, we proceed to extend this result to entire functions, that is, functions holomorphic at every point of $\mathbb{C}^{n}$. For this purpose, one considers entire functions in $\mathbb{R}^{2 n}$ generated by entire functions in $\mathbb{C}^{n}$, that is, real functions $p(\mathbf{x}, \mathbf{y})$ analytic at every point of $\mathbb{R}^{2 n}$ such that there exists an entire function $f(\mathbf{z})$ in $\mathbb{C}^{n}$ and $p(\mathbf{x}, \mathbf{y})=f(\mathbf{x}+i \mathbf{y})$. In this situation, we study the existence of real non-constant factors. Here, "real non-constant factors" means non-unit elements in the ring of real-defined functions, analytic everywhere in $\mathbb{R}^{2 n}$. More precisely, one has the following result.

Proposition 13. Let $f(\mathbf{z})$ be a nonzero entire function in $\mathbb{C}^{n}$, and let $p(\mathbf{x}, \mathbf{y})=$ $f(\mathbf{x}+\mathbf{i} \mathbf{y})$. Then, there exists no decomposition of the form $p(\mathbf{x}, \mathbf{y})=k(\mathbf{x}, \mathbf{y})$. $h(\mathbf{x}, \mathbf{y})$, where $k$ is a non-unit, real-defined function, analytic at every point of $\mathbb{R}^{2 n}$, and $h$ is a complex valued, entire function in $\mathbb{C}^{2 n}$.

Proof. Let us assume that such decomposition $p=k \cdot h$ exists. Then, there is $\mathbf{c} \in \mathbb{R}^{2 n}$ such that $k(\mathbf{c})=0$. We consider now the complex germ $\mathbf{p}$ of $p$ at $\mathbf{c}$, $\mathbf{p} \in \mathcal{O}_{\mathbb{C}^{2 n}, \mathbf{c}}$, and the real germ $\mathbf{k}$ of $k$ at $\mathbf{c}, \mathbf{k} \in \mathcal{O}_{\mathbb{R}^{2 n}, \mathbf{c}}$. Then, since $k$ vanishes at $\mathbf{c}$, it follows that $\mathbf{k}$ is not a unit in $\mathcal{O}_{2 n, \mathbf{c}}^{\mathbb{R}}$, and $\mathbf{k}$ is a factor of $\mathbf{p}$, which is impossible by Proposition 12 .

\section{Non-Standard Cauchy-Riemann Conditions}

In this final section we show how the well known Cauchy-Riemann holomorphic conditions can be generalized for the case of arbitrary finite field extensions, and we deduce some important facts on analytic polynomials.

Definition 2. (See [8]) Let $D=\left(d_{1}, \ldots, d_{2 n-1}\right)$ be a $2 n-1$ tuple. The finite Hankel matrix of order $n$ generated by $D$ is the $n \times n$ matrix $H$ such that $H_{i j}=d_{i+j-1}$.

$$
H=\left(\begin{array}{ccccc}
d_{1} & d_{2} & d_{3} & \ldots & d_{n} \\
d_{2} & d_{3} & & \ldots & d_{n+1} \\
d_{3} & & & & \vdots \\
\vdots & & & & \vdots \\
d_{n} & d_{n+1} & \ldots & \ldots & d_{2 n-1}
\end{array}\right)
$$


Let us introduce $(r+1)$ Hankel, square matrices $\left\{H_{i}\right\}_{i=0, \ldots, r}$, all of them of order $(r+1)$. For this purpose, first, we express $\alpha^{r+i}$ (with $\left.i=1, \ldots, r\right)$ in the $\alpha$-basis, as

$$
\alpha^{r+i}=\sum_{j=0}^{r} a_{i j} \alpha^{j}, \text { with } a_{i j} \in \mathbb{K} \text { and } i=1, \ldots, r \text {. }
$$

Then

- $H_{0}$ is the $(r+1) \times(r+1)$ Hankel matrix generated by the $(2 r+1)$-tuple

$$
\left(1,0 \ldots, 0, a_{10}, \ldots, a_{r 0}\right),
$$

- for $i>1, H_{i}$ is the $(r+1) \times(r+1)$ Hankel matrix generated by the $(2 r+1)$-tuple

$$
(\overbrace{0, \ldots, 0}^{i \text { zeros }}, 1,0, \ldots, 0, a_{1 i}, \ldots, a_{r i}) .
$$

Therefore,

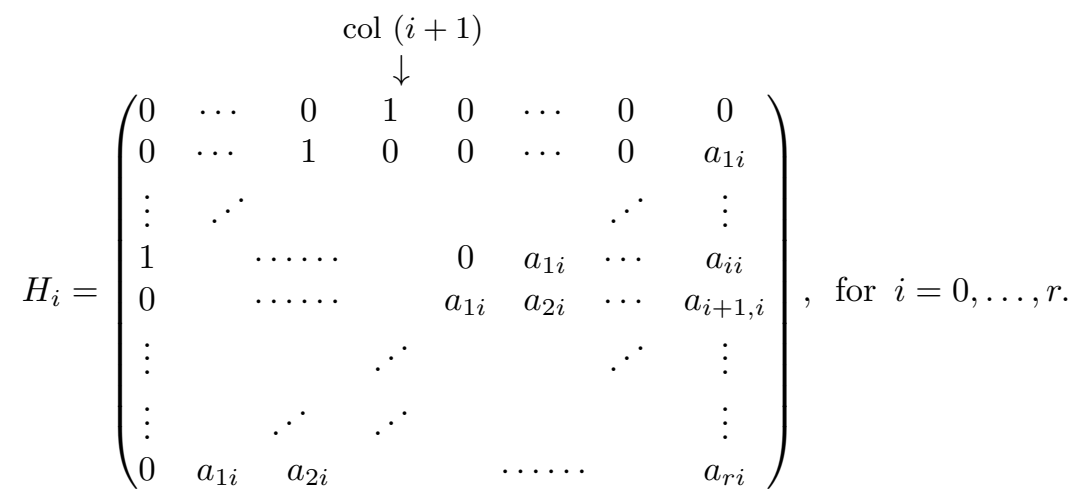

Remark 1. Note that

$$
\left(\begin{array}{cccc}
1 & \alpha & \cdots & \alpha^{r} \\
\alpha & \alpha^{2} & \cdots & \alpha^{r+1} \\
\vdots & & . \cdot & \vdots \\
\alpha^{r} & \alpha^{r+1} & \cdots & \alpha^{2 r}
\end{array}\right)=\sum_{i=0}^{r} \alpha^{i} H_{i}
$$

is the Hankel matrix of order $r+1$ generated by $\left(1, \alpha, \ldots, \alpha^{2 r}\right)$.

In this situation we introduce the following definition

Definition 3. We say that $p(\mathbf{X})=u_{0}(\mathbf{X})+\cdots+u_{r}(\mathbf{X}) \alpha^{r} \in \mathbb{K}(\alpha)[\mathbf{X}]$, with $u_{i} \in \mathbb{K}[\mathbf{X}]$, satisfies the non-standard Cauchy-Riemann conditions (in short: NS(R) if, for $i=0, \ldots, r$, it holds that

$$
\left(\begin{array}{ccc}
\frac{\partial u_{i}}{\partial x_{01}} & \cdots & \frac{\partial u_{i}}{\partial x_{0 n}} \\
\vdots & & \vdots \\
\frac{\partial u_{i}}{\partial x_{r 1}} & \cdots & \frac{\partial u_{i}}{\partial x_{r n}}
\end{array}\right)=H_{i} \cdot\left(\begin{array}{ccc}
\frac{\partial u_{0}}{\partial x_{01}} & \cdots & \frac{\partial u_{0}}{\partial x_{0 n}} \\
\vdots & & \vdots \\
\frac{\partial u_{r}}{\partial x_{01}} & \cdots & \frac{\partial u_{r}}{\partial x_{0 n}}
\end{array}\right)
$$


We will use the following simpler notation for the above conditions:

$$
M_{i}^{L}=H_{i} M^{R}
$$

Example 2. Let $\alpha^{3}+2=0$ (so $r=2$ ) and $n=1$. Let $p\left(x_{01}, x_{11}, x_{21}\right)$ be an $\alpha$-analytic polynomial $p \in \mathbb{Q}(\alpha)\left[x_{01}, x_{11}, x_{21}\right]$, generated by the polynomial $f\left(z_{1}\right)=z_{1}^{2}+1$. Thus

$$
p\left(x_{01}, x_{11}, x_{21}\right)=\left(x_{01}+x_{11} \alpha+x_{21} \alpha^{2}\right)^{2}+1 .
$$

Let $p$ be expressed as

$$
p=u_{0}\left(x_{01}, x_{11}, x_{21}\right)+u_{1}\left(x_{01}, x_{11}, x_{21}\right) \alpha+u_{2}\left(x_{01}, x_{11}, x_{21}\right) \alpha^{2}
$$

where $u_{0}, u_{1}, u_{2}$ are the $\mathbb{Q}$-components of $p$ and

$$
\begin{aligned}
& u_{0}=x_{01}^{2}-4 x_{11} x_{21}+1 \\
& u_{1}=2 x_{01} x_{11}-2 x_{21}^{2} \\
& u_{2}=2 x_{01} x_{21}+x_{11}^{2} .
\end{aligned}
$$

Now, we construct the Hankel matrices $H_{0}, H_{1}, H_{2}$. For this we need to express $\alpha^{3}$ and $\alpha^{4}$ in the $\alpha$-basis:

$$
\begin{aligned}
& \alpha^{3}=a_{10}+a_{11} \alpha+a_{12} \alpha^{2}=-2 \\
& \alpha^{4}=a_{20}+a_{21} \alpha+a_{22} \alpha^{2}=-2 \alpha .
\end{aligned}
$$

Then,

$$
\begin{aligned}
H_{0}=\left(\begin{array}{ccc}
1 & 0 & 0 \\
0 & 0 & a_{10} \\
0 & a_{10} & a_{20}
\end{array}\right)=\left(\begin{array}{ccc}
1 & 0 & 0 \\
0 & 0 & -2 \\
0 & -2 & 0
\end{array}\right) \\
H_{1}=\left(\begin{array}{ccc}
0 & 1 & 0 \\
1 & 0 & a_{11} \\
0 & a_{11} & a_{21}
\end{array}\right)=\left(\begin{array}{ccc}
0 & 1 & 0 \\
1 & 0 & 0 \\
0 & 0 & -2
\end{array}\right) \\
H_{2}=\left(\begin{array}{ccc}
0 & 0 & 1 \\
0 & 1 & a_{12} \\
1 & a_{12} & a_{22}
\end{array}\right)=\left(\begin{array}{ccc}
0 & 0 & 1 \\
0 & 1 & 0 \\
1 & 0 & 0
\end{array}\right)
\end{aligned}
$$

Moreover,

$$
M_{i}^{L}=\left(\begin{array}{c}
\frac{\partial u_{i}}{\partial x_{01}} \\
\frac{\partial u_{i}}{\partial x_{11}} \\
\frac{\partial u_{i}}{\partial x_{21}}
\end{array}\right), M^{R}=\left(\begin{array}{c}
\frac{\partial u_{0}}{\partial x_{01}} \\
\frac{\partial u_{1}}{\partial x_{1}} \\
\frac{\partial u_{2}}{\partial x_{01}}
\end{array}\right)
$$

And one can easily check that $M_{i}^{L}=H_{i} \cdot M^{R}$; compare to Theorem 15 .

Remark 2. Let us verify that we get, in particular, the usual CR conditions from the NS-CR conditions. We consider that $\alpha=i, n=1$ and $r+1=2$. In this context we usually express $z=x+\mathrm{i} y$, but here, following the notation 
introduced for the general case, we will use $\mathbf{X}=\left(\mathbf{x}_{0}, \mathbf{x}_{1}\right)$, with $\mathbf{x}_{0}=\left(x_{01}\right), \mathbf{x}_{1}=$ $\left(x_{11}\right)$. Let $p(\mathbf{X}) \in \mathbb{C}[\mathbf{X}]$ be expressed as

$$
p(\mathbf{X})=u_{0}(\mathbf{X})+\mathbf{i} u_{1}(\mathbf{X}), \text { with } u_{0}, u_{1} \in \mathbb{R}[\mathbf{X}] .
$$

In order to construct the Hankel matrices $H_{i}$, note that $i^{2}=-1$, and thus $a_{10}=-1$, and $a_{11}=0$. Then we have

$$
H_{0}=\left(\begin{array}{rr}
1 & 0 \\
0 & -1
\end{array}\right), H_{1}=\left(\begin{array}{ll}
0 & 1 \\
1 & 0
\end{array}\right)
$$

Therefore the NS-CR conditions are, in this case,

$$
\left(\begin{array}{c}
\frac{\partial u_{0}}{\partial x_{01}} \\
\frac{\partial u_{0}}{\partial x_{11}}
\end{array}\right)=H_{0}\left(\begin{array}{c}
\frac{\partial u_{0}}{\partial x_{01}} \\
\frac{\partial u_{1}}{\partial x_{01}}
\end{array}\right),\left(\begin{array}{c}
\frac{\partial u_{1}}{\partial x_{01}} \\
\frac{\partial u_{1}}{\partial x_{11}}
\end{array}\right)=H_{1}\left(\begin{array}{c}
\frac{\partial u_{0}}{\partial x_{01}} \\
\frac{\partial u_{1}}{\partial x_{01}}
\end{array}\right) .
$$

which yields

$$
\frac{\partial u_{0}}{\partial x_{11}}=-\frac{\partial u_{1}}{\partial x_{01}}, \frac{\partial u_{1}}{\partial x_{11}}=\frac{\partial u_{0}}{\partial x_{01}}
$$

that are the classical CR conditions.

In Lemma 1 we already have a characterization of analytic polynomials. The following theorem characterizes analytic polynomials in terms of the NSCR conditions. First we show that the set of polynomials that satisfy NS-CR is closed under derivation.

Lemma 14. Let $p(\mathbf{X})$ satisfy NS-CR. Then $\frac{\partial p}{\partial x_{i j}}$ also satisfy NS-CR.

Proof. Just take the equation (3) and compute derivatives with respect to $x_{i j}$.

Theorem 15. Let $p(\mathbf{X}) \in \mathbb{K}(\alpha)[\mathbf{X}]$. The following statements are equivalent

1. $p \in \mathbb{A}_{\alpha}[\mathbf{X}]$, generated by some $f(\mathbf{z})$.

2. $p$ satisfies the non-standard Cauchy-Riemann conditions.

Proof. Let us see that (1) implies (2). Let $C_{j}^{i, L}$ and $C_{j}^{R}$ the $j$-column of $M_{i}^{L}$ and $M^{R}$, respectively (see Definition 3). Then, it is enough to prove that $C_{j}^{i, L}=H_{i} C_{j}^{R}$ for $i=0, \ldots, r$ and $j=1, \ldots, n$. For $j=1, \ldots, n$, let $M_{j}$ be the matrix whose columns are $\left(C_{j}^{0, L}, \ldots, C_{j}^{r, L}\right)$; that is

$$
M_{j}=\left(\begin{array}{ccc}
\frac{\partial u_{0}}{\partial x_{0 j}} & \cdots & \frac{\partial u_{r}}{\partial x_{0 j}} \\
\vdots & & \vdots \\
\frac{\partial u_{0}}{\partial x_{r j}} & \cdots & \frac{\partial u_{r}}{\partial x_{r j}}
\end{array}\right)
$$


Computing partial derivatives w.r.t. $x_{0 j}, \ldots, x_{r j}$ in the equality

$$
p(\mathbf{X})=\sum_{i=0}^{r} \alpha^{i} u_{i}(\mathbf{X})=f\left(\sum_{i=0}^{r} \alpha^{i} \mathbf{x}_{i}\right)
$$

one gets that

$$
M_{j} \cdot\left(\begin{array}{c}
1 \\
\vdots \\
\alpha^{r}
\end{array}\right)=\frac{\partial f}{\partial z_{j}}\left(\sum_{i=0}^{r} \alpha^{i} \mathbf{x}_{i}\right)\left(\begin{array}{c}
1 \\
\vdots \\
\alpha^{r}
\end{array}\right)
$$

Now, from the equality

$$
\frac{\partial f}{\partial z_{j}}\left(\sum_{i=0}^{r} \alpha^{i} \mathbf{x}_{i}\right)=\frac{\partial p}{\partial x_{0 j}}=\sum_{i=0}^{r} \frac{\partial u_{i}}{\partial x_{0 j}} \alpha^{i}
$$

one obtains that $\sum_{i=0}^{r} \alpha^{i} C_{j}^{i, L}=$

$M_{j} \cdot\left(\begin{array}{c}1 \\ \vdots \\ \alpha^{r}\end{array}\right)=\left(\begin{array}{cccc}1 & \alpha & \cdots & \alpha^{r} \\ \alpha & \alpha^{2} & \cdots & \alpha^{r+1} \\ \vdots & & . \cdot & \vdots \\ \alpha^{r} & \alpha^{r+1} & \cdots & \alpha^{2 r}\end{array}\right) \cdot\left(\begin{array}{c}\frac{\partial u_{0}}{\partial x_{0 j}} \\ \vdots \\ \frac{\partial u_{r}}{\partial x_{0 j}}\end{array}\right)=\left(\begin{array}{cccc}1 & \alpha & \cdots & \alpha^{r} \\ \alpha & \alpha^{2} & \cdots & \alpha^{r+1} \\ \vdots & & . & \vdots \\ \alpha^{r} & \alpha^{r+1} & \cdots & \alpha^{2 r}\end{array}\right) \cdot C_{j}^{R}$

Thus, by Remark 1, we get

$$
\sum_{i=0}^{r} \alpha^{i} C_{j}^{i, L}=\sum_{i=0}^{r} \alpha^{i} H_{i} \cdot C_{j}^{R}
$$

Therefore, since $C_{j}^{i, L}, C_{j}^{R}$ are over $\mathbb{K}[\mathbf{X}]$, and $H_{i}$ is over $\mathbb{K}$, we get

$$
C_{j}^{i, L}=H_{i} \cdot C_{j}^{R}
$$

concluding the proof of this implication.

Let us see that (2) implies (1). We express $p(\mathbf{X})$ as $p(\mathbf{X})=p^{m}(\mathbf{X})+\cdots+p^{0}(\mathbf{X})$, where $p^{k}(\mathbf{X})(k \in\{0, \ldots, m\}$, being $m$ the total degree of $p)$ is the homogeneous component of degree $k$ of $p(\mathbf{X})$; i.e. in $p^{k}(\mathbf{X})$, all terms of $p(\mathbf{X})$ of total degree $k$ are collected. Now, each homogeneous part $p^{k}(\mathbf{X})$ is written as

$$
p^{k}(\mathbf{X})=u_{0}^{k}(\mathbf{X})+\cdots+\alpha^{r} u_{r}^{k}(\mathbf{X})
$$

where $u_{j}^{k} \in \mathbb{K}[\mathbf{X}]$ is homogeneous of degree $k$. Obviously, each component of $p$ verifies

$$
u_{i}=u_{i}^{m}+\cdots+u_{i}^{0} .
$$

Now, taking into account that the partial derivative of a homogeneous polynomial is again homogeneous and that two polynomials in $\mathbb{K}[\mathbf{X}]$ are equal iff their homogeneous components are equal, we deduce that the NS-CR conditions are 
also valid if we replace $u_{j}$ by $u_{j}^{k}$; that is $p^{k}$ satisfies also the NS-CR conditions. So, our plan now is to prove that each $p^{k}$ is analytic, from where one deduces that $p$ is analytic. We will do it by induction in the degree, being the result trivial if $k=0$, since every constant polynomial is $\alpha$-analytic. From the NS-CR conditions, we get that for $j=1, \ldots, n$, and $i=0, \ldots, r$,

$$
\left(\begin{array}{c}
\frac{\partial u_{i}^{k}}{\partial x_{0 j}} \\
\vdots \\
\frac{\partial u_{i}^{k}}{\partial x_{r j}}
\end{array}\right)=H_{i} \cdot\left(\begin{array}{c}
\frac{\partial u_{0}^{k}}{\partial x_{0 j}} \\
\vdots \\
\frac{\partial u_{r}^{k}}{\partial x_{0 j}}
\end{array}\right)
$$

Thus, by Remark 1, we obtain, for $j=1, \ldots, n$,

$$
\left(\begin{array}{c}
\frac{\partial p^{k}}{\partial x_{0 j}} \\
\vdots \\
\frac{\partial p^{k}}{\partial x_{r j}}
\end{array}\right)=\left(\begin{array}{cccc}
1 & \alpha & \cdots & \alpha^{r} \\
\alpha & \alpha^{2} & \cdots & \alpha^{r+1} \\
\vdots & & . \cdot & \vdots \\
\alpha^{r} & \alpha^{r+1} & \cdots & \alpha^{2 r}
\end{array}\right) \cdot\left(\begin{array}{c}
\frac{\partial u_{0}^{k}}{\partial x_{0 j}} \\
\vdots \\
\frac{\partial u_{r}^{k}}{\partial x_{0 j}}
\end{array}\right)=\frac{\partial p^{k}}{\partial x_{0 j}}\left(\begin{array}{c}
1 \\
\vdots \\
\alpha^{r}
\end{array}\right)
$$

Now, applying Euler's formula and the previous identities, we get

$$
p^{k}=\frac{1}{k}\left(\left(x_{01}+\cdots+\alpha^{r} x_{r 1}\right) \frac{\partial p^{k}}{\partial x_{01}}+\cdots+\left(x_{0 n}+\cdots+\alpha^{r} x_{r n}\right) \frac{\partial p^{k}}{\partial x_{0 n}}\right) .
$$

The partial derivative $\frac{\partial p^{k}}{\partial x_{0 i}}$ is a homogeneous polynomial of degree $<k$ that satisfies NS-CR, so, by induction hypothesis, $\frac{\partial p^{k}}{\partial x_{0 i}}$ is $\alpha$-analytic generated by $f_{i}(\mathbf{z})$. It follows that $p^{k}$ is $\alpha$-analytic generated by $(1 / k)\left(z_{1} f_{1}(\mathbf{z})+\ldots+z_{n} f_{n}(\mathbf{z})\right)$.

In Corollary 2, we have seen that an analytic polynomial is constant if and only if its generator is constant. In the complex case, an important application of the CR condition is that an analytic polynomial is constant if and only if either the real or the imaginary part is constant; that is, it is enough to check whether one of its component is constant. In the following we show that our NS-CR conditions yield to the same conclusion. For this purpose, we first state two technical lemmas.

Lemma 16. Let $H$ be the $n \times n$ Hankel matrix, $n>1$, generated by $\left(d_{1}, \ldots, d_{2 n-1}\right) \in$ $\mathbb{K}^{2 n-1}$, and let $\left(b_{0}, \ldots, b_{n-1}\right) \in \mathbb{K}^{n}$ be such that

$$
H \cdot\left(\begin{array}{c}
b_{0} \\
\vdots \\
b_{n-2} \\
b_{n-1}
\end{array}\right)=\left(\begin{array}{c}
d_{n+1} \\
\vdots \\
d_{2 n-1} \\
\sum_{i=0}^{n-1} d_{n+i} b_{i}
\end{array}\right) .
$$

If $f(t)=t^{n}-b_{n-1} t^{n-1}-\cdots-b_{0} \in \mathbb{K}[t]$ is irreducible over $\mathbb{K}$, then $\operatorname{det}(H) \neq 0$. 
Proof. Let

$$
g(t)=d_{1} t^{n-1}+\left(d_{2}-d_{1} b_{n-1}\right) t^{n-2}+\cdots+\left(d_{n}-d_{n-1} b_{n-1}-\cdots-d_{1} b_{1}\right) .
$$

We consider the rational function $R(t)=\frac{g}{f}$. Then, $H$ is the $n \times n$ principal matrix of the infinity Hankel matrix associated to $R(t)$ (see the concept of associated matrix in [8] Def. 7.2.3). Since $\operatorname{deg}(g)<\operatorname{deg}(f)$, and $f$ is irreducible, then $R(t)=\frac{g}{f}$ is a proper representation. Now, the result follows from Theorem 7.2.2. and 7.3.1. in [8], that states that the rank of the infinite matrix associated to a properly expressed rational function agrees with the degree of the denominator.

Lemma 17. $\operatorname{det}\left(H_{i}\right) \neq 0$.

Proof. Let $m(t)=t^{r+1}-b_{r} t^{r}-\cdots-b_{1} t-b_{0}$ be the minimal polynomial of $\alpha$. Let $\alpha^{2 r+1}$ be expressed in the $\alpha$-basis as

$$
\alpha^{2 r+1}=a_{(r+1) 0}+a_{(r+1) 1} \alpha+\cdots+a_{(r+1) r} \alpha^{r} .
$$

Then

$$
\left(\begin{array}{cccc}
1 & \alpha & \cdots & \alpha^{r} \\
\alpha & \alpha^{2} & \cdots & \alpha^{r+1} \\
\vdots & & . \cdot & \vdots \\
\alpha^{r} & \alpha^{r+1} & \cdots & \alpha^{2 r}
\end{array}\right)\left(\begin{array}{c}
b_{0} \\
\vdots \\
b_{r}
\end{array}\right)=\left(\begin{array}{c}
\alpha^{r+1} \\
\vdots \\
\alpha^{2 r+1}
\end{array}\right)
$$

Thus, by Remark 1,

$$
\left(\begin{array}{c}
\alpha^{r+1} \\
\vdots \\
\alpha^{2 r+1}
\end{array}\right)=\sum_{i=0}^{r} \alpha^{i}\left(\begin{array}{c}
a_{1 i} \\
\vdots \\
a_{r i} \\
a_{(r+1) i}
\end{array}\right)=\sum_{i=0}^{r} \alpha^{i} H_{i} \cdot\left(\begin{array}{c}
b_{0} \\
\vdots \\
b_{r}
\end{array}\right) .
$$

Hence, taking into account that $a_{i j}, b_{k} \in \mathbb{K}$ one deduces that

$$
H_{i} \cdot\left(\begin{array}{c}
b_{0} \\
\vdots \\
b_{r}
\end{array}\right)=\left(\begin{array}{c}
a_{1 i} \\
\vdots \\
a_{r i} \\
a_{(r+1) i}
\end{array}\right)
$$

We consider

$$
\left\{\begin{array}{ccc}
\alpha^{r} & = & \alpha^{r} \\
\alpha^{r+1} & = & a_{10}+a_{11} \alpha+\cdots+a_{1 r} \alpha^{r} \\
& \vdots & \\
\alpha^{2 r} & = & a_{r 0}+a_{r 1} \alpha+\cdots+a_{r r} \alpha^{r}
\end{array}\right.
$$


Multiplying the $i$-th equality by $b_{i-1}$, adding all of them, and taking into account that $a_{i j}, b_{k} \in \mathbb{K}$, we get

$$
\begin{aligned}
a_{10} b_{1}+\cdots+a_{r 0} b_{r} & =a_{(r+1) 0} \\
& \vdots \\
a_{1(r-1)} b_{1}+\cdots+a_{r(r-1)} b_{r} & =a_{(r+1)(r-1)} \\
b_{0}+a_{1 r} b_{1}+\cdots+a_{r r} b_{r} & =a_{(r+1) r}
\end{aligned}
$$

Therefore, we get that for $i=0, \ldots, r$, the the last row of $H_{i}$ times the column $\left(b_{0}, \ldots, b_{r}\right)^{T}$ is equal to $a_{(r+1) i}$. Now, the result follows from Lemma 16.

Theorem 18. An analytic polynomial $p$ is constant if and only if at least one of its $\mathbb{K}$-components is constant.

Proof. If $p$ is constant it is clear that all $\mathbb{K}$-components are constant. Conversely, let $u_{0}, \ldots, u_{r}$ be the $\mathbb{K}$-components of $p$, and let $u_{i}$ be a constant. By Theorem 15, the NS-CR conditions are satisfied; let us denote them by $M_{i}^{L}=H_{i} M^{R}$. Now, by Lemma $17, H_{i}^{-1} M_{i}^{L}=M^{R}$. Moreover, since $u_{i}$ is constant, then $M_{i}^{L}$ is the zero matrix, and hence $M^{R}$ is also zero. Now, coming back to the NS-CR conditions we get that $M_{j}^{L}$ is the zero matrix for $j=0, \ldots, r$. That is, all $u_{j}$ are constant.

Remark 3. Note that Corollary 4 can also be proved directly from Theorem 18. If $\mathbf{p}$ is an $\alpha$-analytic polynomial and $\mathbf{p} \in \mathbb{K}[\mathbf{X}]$, then its component associated to $\alpha$ is constant (in fact, is zero), so $\mathbf{p}$ is a constant polynomial.

Remark 4. Throughout the text, we have assumed that $\mathbb{K}$ is a characteristic zero field. The results obtained cannot be extended to characteristic $p$ fields. For instance, if $\mathbb{K}$ is a characteristic $p$ field and $\mathbb{K}(\alpha)$ is an extension of degree $r+1$ (separable or not), take $n=1$ and $x_{01}^{p} \in \mathbb{K}(\alpha)\left[x_{01}, \ldots, x_{r 1}\right]$. Clearly, $x_{01}^{p}$ verifies the NS-CR but it is not $\alpha$-analytic. If it were, since the transformation in Equation (1) is homogeneous, $x_{01}$ would be generated by a polynomial of the form $f\left(z_{1}\right)=k \cdot z_{1}^{p}, k \in \overline{\mathbb{K}}$. But, in this case, $x_{01}^{p}=k\left(x_{01}+\alpha x_{11}+\cdots+\alpha^{r} x_{r 1}\right)^{p}=$ $k x_{01}^{p}+k \alpha^{p} x_{11}^{p}+\cdots+k \alpha^{p r} x_{r 1}^{p}$, which is impossible.

\section{Acknowledgements}

The authors are partially supported by the Spanish Ministerio de Ciencia e Innovación, Ministerio de Economía y Competitividad and by the European Regional Development Fund (ERDF), under the projects MTM2008-04699-C03-(01,03) and MTM2011-25816-C02-(01,02). Second and fourth authors belong to the Research Group ASYNACS (Ref. CCEE2011/R34).

We thank the anonymous referees for many helpful suggestions. 


\section{References}

[1] C. Andradas, T. Recio, J. R. Sendra, Base field restriction techniques for parametric curves, in: Proceedings of the 1999 International Symposium on Symbolic and Algebraic Computation (Vancouver, BC), ACM, New York, 1999, pp. 17-22 (electronic).

[2] J. Bak, D. Newman, Complex analysis, Undergraduate texts in mathematics, Springer, 1997.

[3] T. Recio, J. R. Sendra, Real reparametrizations of real curves, J. Symbolic Comput. 23 (2-3) (1997) 241-254, parametric algebraic curves and applications (Albuquerque, NM, 1995).

[4] T. Recio, J. R. Sendra, L. F. Tabera, C. Villarino, Generalizing circles over algebraic extensions, Math. Comp. 79 (270) (2010) 1067-1089.

[5] W. Rudin, Real and complex analysis, 3rd ed., McGraw-Hill, Inc., New York, NY, USA, 1987.

[6] J. R. Sendra, Hankel matrices and computer algebra, SIGSAM Bull. 24 (1990) 17-26.

[7] J. R. Sendra, C. Villarino, Optimal reparametrization of polynomial algebraic curves, Internat. J. Comput. Geom. Appl. 11 (4) (2001) 439-453.

[8] F. Winkler, Polynomial Algorithms in Computer Algebra, Springer-Verlag New York, Inc., Secaucus, NJ, USA, 1996.

[9] O. Zariski, P. Samuel, Commutative algebra. Vol. I, Springer-Verlag, New York, 1975, with the cooperation of I. S. Cohen, Graduate Texts in Mathematics, No. 28. 